\title{
Phase transition, crystal structure, and magnetic order in VOCI
}

\author{
Andreas Schönleber, ${ }^{1}$ Joachim Angelkort, ${ }^{1}$ Sander van Smaalen,,${ }^{1 *}$ Lukáš Palatinus, ${ }^{2}$ \\ Anatoliy Senyshyn, ${ }^{3, \dagger}$ and Wolfgang Morgenroth ${ }^{4, \hbar}$ \\ ${ }^{1}$ Laboratory of Crystallography, University of Bayreuth, 95440 Bayreuth, Germany \\ ${ }^{2}$ Laboratory of Crystallography, EPF Lausanne, 1015 Lausanne, Switzerland \\ ${ }^{3}$ FRM II-TUM, 85747 Garching, Germany \\ ${ }^{4}$ Laboratoy of Earth Science, University of Frankfurt, 60438 Frankfurt am Main, Germany \\ (Received 3 April 2009; revised manuscript received 1 July 2009; published 31 August 2009)
}

\begin{abstract}
VOCl develops magnetic order below $T_{\mathrm{N}}=80.3(3) \mathrm{K}$. Employing single-crystal x-ray diffraction and powder neutron diffraction it is shown that the magnetic phase transition is accompanied by a small, temperaturedependent monoclinic lattice distortion with $\gamma=90.212(2)^{\circ}$ at $T=3.2 \mathrm{~K}$. The phase transition presumably is of second order with a critical exponent $\beta=0.099(12)$. The monoclinic distortion lifts the frustration of interchain interactions along $\frac{1}{2} \mathbf{a} \pm \frac{1}{2} \mathbf{b}$ of the orthorhombic room-temperature structure with the result that chains of antiferromagnetic order exist along $\frac{1}{2} \mathbf{a}+\frac{1}{2} \mathbf{b}$ while ferromagnetic chains exist along $\frac{1}{2} \mathbf{a}-\frac{1}{2} \mathbf{b}$. Both antiferromagnetic interchain coupling (if stronger along $\frac{1}{2} \mathbf{a}+\frac{1}{2} \mathbf{b}$ than along $\frac{1}{2} \mathbf{a}-\frac{1}{2} \mathbf{b}$ ) and ferromagnetic interchain coupling (if stronger along $\frac{1}{2} \mathbf{a}-\frac{1}{2} \mathbf{b}$ than along $\frac{1}{2} \mathbf{a}+\frac{1}{2} \mathbf{b}$ ) can explain the observed magnetic superstructure with magnetic space group $F 2^{\prime} / d^{\prime}$ on the $2 a \times 2 b \times 2 c$ supercell (standard setting $C 2^{\prime} / c^{\prime}$ ) and with magnetic moments parallel to a. Remarkably, the lattice distortion is not accompanied by a structural distortion so that the driving force for the lattice distortion is completely governed by the magnetic interactions.
\end{abstract}

DOI: 10.1103/PhysRevB.80.064426

PACS number(s): 61.50.Ks, 61.66.Fn, 75.25.+z, 75.30.Et

\section{INTRODUCTION}

Isostructural compounds $\mathrm{TiO} X(X=\mathrm{Cl}$ and $\mathrm{Br})$ have recently been studied for their properties related to the presence of quasi-one-dimensional $S=1 / 2$ magnetic chains of $\mathrm{Ti}$ atoms. ${ }^{1}$ Upon cooling, TiOCl undergoes a phase transition at $T_{c 2}=90 \mathrm{~K}$ toward an incommensurately modulated state. ${ }^{2,3}$ At $T_{c 1}=67 \mathrm{~K}$ a first-order, lock-in transition occurs toward a twofold superstructure that has been interpreted as a spinPeierls state. ${ }^{1,4}$ Both transitions are accompanied by anomalies in the magnetic susceptibility which indicate the development of magnetic order. $\mathrm{TiOBr}$ exhibits a similar series of phase transitions. ${ }^{5}$

Other compounds isostructural to $\mathrm{TiOCl}$ include $\mathrm{VOCl}$, $\mathrm{CrOCl}$, and $\mathrm{FeOCl} .6,7$ They differ from $\mathrm{TiOCl}$ in the number of $d$ electrons on the metal atom. The properties of $\mathrm{TiOCl}$ were found to critically depend on the presence of the single $d$ electron of $\mathrm{Ti}^{3+}$ in the $3 d_{x^{2}-y^{2}}$ orbital, which gives rise to a large direct exchange along the chains of metal atoms and which allows only weak magnetic exchange between the chains. ${ }^{8}$ Other compounds $\mathrm{MOCl}$ contain $M^{3+}$ ions with more than one $3 d$ electrons thus requiring other $3 d$ orbitals to be filled too. For example, vanadium in $\mathrm{VOCl}$ has two $3 d$ electrons resulting in a magnetic moment of $S=1$. With two filled $3 d$ orbitals $\mathrm{VOCl}$ has strong interchain coupling thus forcing a different phase diagram than $\mathrm{TiOCl}$ and $\mathrm{TiOBr}$ compounds as has been observed experimentally indeed.

In $\mathrm{VOCl}$ one phase transition has been found in the temperature-dependent magnetic susceptibility, at $T_{\mathrm{N}}$ $=80.5(5) \mathrm{K}$. Neutron diffraction indicated that the lowtemperature phase corresponds to a twofold magnetic superstructure of antiferromagnetic order. ${ }^{9}$ At that time a complicated two-phase structure model was proposed that has orthorhombic lattice symmetry. ${ }^{9}$ Here we show that the symmetry of the low-temperature phase of $\mathrm{VOCl}$ is monoclinic and we provide an alternative proposal for the magnetic superstructure that is based on this symmetry. A remarkable property of this phase transition is that the monoclinic lattice distortion is not associated with distortions of the nuclear structure. The sole driving force for the lattice distortion must thus come from the magnetic interactions.

\section{EXPERIMENTAL}

\section{A. Synthesis and crystal structure}

Single crystals and powder sample of $\mathrm{VOCl}$ were grown by gas transport following a published procedure. ${ }^{10}$ The educts $\mathrm{VO}_{2}$ (Aldrich, 99.9\% purity) and $\mathrm{VCl}_{3}$ (Alpha, 99\% purity) were placed as a stoichiometric mixture in a sealed and evacuated quartz glass tube $\left(p=6 \times 10^{-3} \mathrm{kPa}\right)$, which was then heated in a temperature gradient of 923-823 K for 135 h. Transparent, light-brown crystals of $\mathrm{VOCl}$ had a needle-to-platelike habit and were stable in air for several days.

Diffraction at room temperature confirmed the $\mathrm{FeOCl}$ structure type. ${ }^{6,7}$ This structure is built of a stacking of slabs perpendicular to the c axis. Each slab consists of a $M_{2} \mathrm{O}_{2}$ bilayer enclosed by layers of $\mathrm{Cl}$ atoms. The interaction between the slabs is of the van der Waals type. The symmetry is orthorhombic with space group Pmmn. ${ }^{6,7}$ Lattice parameters of $\mathrm{VOCl}$ have been determined at $T=120 \mathrm{~K}$ as $a$ $=3.76837(5), b=3.29445(4)$ and $c=7.8993(2) \AA$, in good agreement with published values.

\section{B. Synchrotron single-crystal $x$-ray diffraction}

Single-crystal x-ray diffraction with synchrotron radiation was measured down to $T=20 \mathrm{~K}$ on beamline D3 at Hasylab, DESY (Hamburg, Germany). A platelike single crystal of 


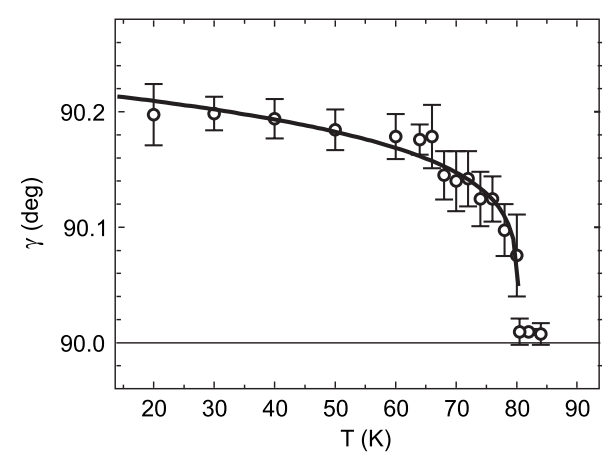

FIG. 1. Temperature dependence of the monoclinic angle $\gamma$ of VOCl. The curve represents a fit to the data according to Eq. (1).

dimensions of approximately $0.1 \times 0.02 \times 0.001 \mathrm{~mm}^{3}$ was glued to a carbon fiber that was attached to a closed-cycle helium cryostat mounted on a Huber four-circle diffractometer with Eulerian cradle. The diffracted intensities were collected with a point detector at the wavelength $\lambda=0.5 \AA$.

For a data collection at $T=20 \mathrm{~K}$ the integrated intensities of reflections were measured by $\omega$ scans centered at the expected reflection positions of the orthorhombic lattice. Owing to the presence of the cryostat, only a limited range of setting angles of the crystal could be reached. Nevertheless, an almost complete data set could be obtained up to $\sin (\theta) / \lambda=0.70 \AA^{-1}$ for the orthorhombic $\mathrm{mmm}$ symmetry. A good fit to the diffraction data has been obtained for the orthorhombic structure model as is also valid at room temperature. ${ }^{11}$ Superlattice reflections have not been found in the x-ray diffraction, in particular they have not been found at the positions $(1 / 2,1 / 2,1 / 2)$.

In a second experiment at $T=20 \mathrm{~K}, 2 \theta$ scans with narrow detector slits were performed on 16 selected reflections. Reflections with both $h \neq 0$ and $k \neq 0$ were split, i.e., they showed a double maximum. (A detailed description of the procedure of $2 \theta$ scans with narrow detector slits is given in Ref. 12.) Similar scans were performed on the reflections $(4, \pm 2,0)$ and $(2, \pm 4,0)$ at selected temperatures within the range $20 \mathrm{~K} \leq T \leq 85 \mathrm{~K}$. The magnitude of the splitting appeared to depend on temperature and it was zero for $T$ $>80 \mathrm{~K}$.

These observations can be explained by a twinned crystal of monoclinic symmetry whereby the two domains give rise to Bragg reflections at the same crystal orientation but at different scattering angles. Since reflections $(0,0, l)$ are not split, the monoclinic axis is the $\mathbf{c}$ axis. The monoclinic angle $\gamma$ was derived from the magnitude of the split of the reflections (Fig. 1).

Its temperature dependence can be described by the power law

$$
\gamma=\gamma_{0}+A^{2}\left(1-T / T_{\mathrm{N}}\right)^{2 \beta}
$$

with $\gamma_{0}=90^{\circ}$ is the orthorhombic angle of the roomtemperature phase. A fit of Eq. (1) to the data below $T$ $=80 \mathrm{~K}$ resulted in a critical exponent of $\beta=0.099(12)$ and a transition temperature $T_{\mathrm{N}}=80.3(3) \mathrm{K}$ while $A=0.471(8)$. Above $T_{\mathrm{N}}$ reflections are not split and $\mathrm{VOCl}$ is in its orthorhombic phase. The orthorhombic-to-monoclinic phase tran- sition at $T_{\mathrm{N}}=80.3(3) \mathrm{K}$ thus coincides with the previously reported phase transition toward a magnetic superstructure. ${ }^{9}$

\section{Neutron-powder diffraction}

Neutron-powder diffraction was measured on the Structure Powder Diffractometer (SPODI) at the research reactor FRM-II (Garching, Germany). ${ }^{13}$ Monochromatic neutrons $(\lambda=1.5481 \AA)$ were obtained at a $155^{\circ}$ take off using the (551) reflection of a vertically focused composite Ge monochromator. The vertical position-sensitive multidetector (300-mm effective height) consisting of $80{ }^{3} \mathrm{He}$ tubes was used for data collection in the $0 \leq 2 \theta \leq 136^{\circ}$ range. The sample was filled into a thin-walled $(0.15 \mathrm{~mm})$ vanadium can of diameter $10 \mathrm{~mm}$ and then mounted in the top-loading closed-cycle refrigerator. Helium-4 was used as a heat transmitting medium. The instantaneous temperature was measured using two Cernox ${ }^{\mathrm{TM}}$ thin-film resistance cryogenic temperature sensors and controlled by a temperature controller from LakeShore ${ }^{\mathrm{TM}}$. Two-dimensional powder-diffraction data were collected at the three temperatures $T=120,20$, and $3.2 \mathrm{~K}$.

Rietveld refinements of the nuclear and magnetic structures were done with the computer program JANA2006. ${ }^{14}$ Background scattering was described by Legendre polynomials. The peak shape was modeled by pseudo-Voigt functions. Angular ranges $38.32^{\circ} \leq 2 \theta \leq 39.22^{\circ}, 56.35^{\circ} \leq 2 \theta$ $\leq 57.25^{\circ}$, and $61.95^{\circ} \leq 2 \theta \leq 62.85^{\circ}$ were excluded from the refinements because they contained reflections from aluminum parts of the cryostat. Data reduction strongly reduced the asymmetry of the diffraction peaks as they are visible in the raw data.

The quality of the data suffers from strong preferred orientation which is the result of the plateletlike shapes of the crystallites that comprise the sample. This has been successfully accounted for by a correction after March Dollase in the Rietveld refinement. The good quality of the fit of the nuclear structure is illustrated by the Rietveld plot for the $T=120 \mathrm{~K}$ data (Fig. 2).

Rietveld refinement of the $T=120 \mathrm{~K}$ data confirmed the orthorhombic crystal structure of the high-temperature phase of VOCl. Rietveld refinement of the low-temperature structures at $T=20 \mathrm{~K}$ and $T=3.2 \mathrm{~K}$ required a monoclinic lattice with a monoclinic angle in agreement with the angle derived from single-crystal x-ray diffraction (TABLE I). A deviation in the atomic parameters from orthorhombic symmetry was not found. This implies that the distortion represents a simple shearing of the lattice without an accompanying structural distortion.

\section{MAGNETIC SUPERSTRUCTURE}

Magnetic superlattice reflections were found in the neutron-powder diffraction measured at $T=20 \mathrm{~K}$ and $T$ $=3.2 \mathrm{~K}$ (Fig. 3). They can be indexed with a modulation wave vector of $\mathbf{q}=\left(\frac{1}{2}, \frac{1}{2}, \frac{1}{2}\right)$ describing the formation of a twofold magnetic superstructure. These observations corroborate those of Wiedenmann et al. ${ }^{9}$

Based on their magnetic neutron-diffraction data, Wiedenmann et al. ${ }^{9}$ have proposed a complicated two-phase model 


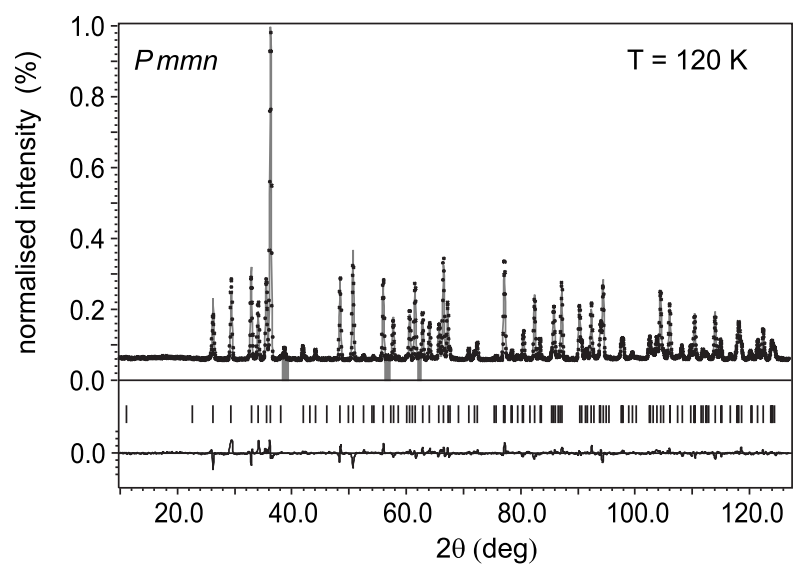

FIG. 2. Rietveld plot of the neutron powder-diffraction pattern of $\mathrm{VOCl}$ at $T=120 \mathrm{~K}$ according to Rietveld refinement in space group Pmmn. The upper trace shows the observed (crosses) and calculated (solid line) intensity profiles; the bottom trace gives the difference between observed and calculated intensities. Vertical bars indicate the positions of the structural Bragg reflections. Gray regions have been excluded from refinement. The fit converged to $R_{\text {Bragg }}=0.037$ and $R_{p}=0.038$.

for the magnetic superstructure on an orthorhombic lattice. Presently we have found that the magnetic phase transition is accompanied by a monoclinic lattice distortion. The correct symmetry for the magnetic superstructure thus is monoclinic and we present here a model with this symmetry.

The nuclear structure is described by space group $P 2 / n$ (c unique) that is a monoclinic subgroup of Pmmn of highest symmetry. The magnetic unit cell is an $F$-centered $2 \mathbf{a} \times 2 \mathbf{b}$ $\times 2$ c supercell of the nuclear structure. The twofold rotation axis of the nuclear structure induces either 2 or $2^{\prime}$ as magnetic symmetry element. The magnetic moment of vanadium is restricted by symmetry to $\left(0,0, M_{z}\right)$ for the magnetic space group $F 2$ of the supercell and it is restricted to $\left(M_{x}, M_{y}, 0\right)$ for $F 2^{\prime}$. Both space groups comprise four symmetryindependent vanadium atoms in the supercell. The $n$ glide of the basic-structure cell becomes a $d$ glide in the $F$-centered cell that pairwise relates atoms which were independent in $F 2$, such that possible magnetic space groups are $F 2^{\prime} / d^{\prime}$, $F 2^{\prime} / d, F 2 / d^{\prime}$, and $F 2 / d$. Standard settings according to Litvin $^{15}$ are $C 2^{\prime} / c^{\prime}, C 2^{\prime} / c, C 2 / c^{\prime}$, and $C 2 / c$, respectively. As can be seen in Fig. 3 and as described in detail below, the best fits to the neutron-diffraction data are achieved with the magnetic space groups $F 2^{\prime} / d$ and $F 2^{\prime} / d^{\prime}$, i.e., with the magnetic moments of vanadium parallel to the layers $\mathrm{VOCl}$, $\left(M_{x}, M_{y}, 0\right)$. The fit could not be improved by lowering the symmetry to $F 2^{\prime}$ or $F d^{\prime}$.
Rietveld refinements of the combined magnetic/nuclear superstructure were performed with the software JANA2006. ${ }^{14}$ Deviations could not be found of the nuclear structure from the Pmmn high-temperature structure. The orthorhombic structure reduced to $P 2 / n$ of the nuclear unit cell is still composed of three independent atoms with their $z$ coordinates as free parameters. The only additional parameters are off-diagonal components of the temperature tensor. However, refinement of these or a reduction in the symmetry to acentric monoclinic space groups lead to highly correlated parameters while refined values of the parameters still obeyed Pmmn symmetry within standard deviations. Additional restrictions according to Pmmn was therefore applied to the atomic-displacement parameters.

High correlations were also encountered between the parameters describing the magnetic moments of the two independent vanadium atoms within each of the four centrosymmetric magnetic space groups. Under the assumption that the phase transition is governed by one order parameter, a structure model was developed within the superspace approach. ${ }^{16}$ The magnetic superstructure then is described by the $\mathbf{a} \times \mathbf{b}$ $\times$ c basic-structure unit cell containing one crystallographically independent atom each of $\mathrm{V}, \mathrm{O}$, and $\mathrm{Cl}$. The magnetic moment of $\mathrm{V}$ is described by a single-harmonic modulation function. The supercell description can be derived from the superspace model by a unique recipe. ${ }^{16}$ It appears that the superspace group $P 2 / n^{\prime}\left(\sigma_{1}, \sigma_{2}, \frac{1}{2}\right) \overline{1} 0$ with $\sigma_{1}=\sigma_{2}=\frac{1}{2}$ leads to supercell symmetry $F 2^{\prime} / d^{\prime}$ and $P 2 / n\left(\sigma_{1}, \sigma_{2}, \frac{1}{2}\right) \overline{1} 0$ leads to supercell symmetry $F 2^{\prime} / d$. A single-harmonic modulation in the superspace model corresponds to the nonspace-group restriction on the ordered magnetic moments of the two independent atoms V1 and V2 in the supercell of

$$
\left[M_{x}(\mathrm{~V} 2), M_{y}(\mathrm{~V} 2), 0\right]=\left[-M_{x}(\mathrm{~V} 1),-M_{y}(\mathrm{~V} 1), 0\right] .
$$

For $P 2^{\prime} / n\left(\sigma_{1}, \sigma_{2}, \frac{1}{2}\right) \overline{1} 0$ with supercell symmetry $F 2 / d$ and for $P 2^{\prime} / n^{\prime}\left(\sigma_{1}, \sigma_{2}, \frac{1}{2}\right) \overline{1} 0$ with supercell symmetry $F 2 / d^{\prime}$ the additional restriction is $\left[0,0, M_{z}(\mathrm{~V} 2)\right]=\left[0,0,-M_{z}(\mathrm{~V} 1)\right]$.

Successful Rietveld refinements of the magnetic superstructure could therefore be performed within $F 2^{\prime} / d$ and $F 2^{\prime} / d^{\prime}$ together with the additional restriction according to Eq. (2). Rietveld refinements were also successful for $F 2 / d$ and $F 2 / d^{\prime}$ together with its appropriate superspace relation between the magnetic moments of the two independent vanadium atoms. The fit to the neutron-powder-diffraction data is similar for the four symmetries, with $R_{p}=0.0507$ and $R_{\text {Bragg }}=0.0707$ for $F 2^{\prime} / d^{\prime}, R_{p}=0.0510$ and $R_{\text {Bragg }}=0.0711$ for $F 2^{\prime} / d, R_{p}=0.0515$ and $R_{\text {Bragg }}=0.0716$ for $F 2 / d^{\prime}$ and $R_{p}=0.0512$ and $R_{\text {Bragg }}=0.0715$ for $F 2 / d$. Almost equal $R$

TABLE I. Lattice parameters of $\mathrm{VOCl}$ as function of temperature, obtained by Rietveld refinement against neutron powder-diffraction data.

\begin{tabular}{rccccc}
\hline \hline \multicolumn{1}{c}{$T$} & $a$ & $b$ & $c$ & $\gamma$ & $V$ \\
$(\mathrm{~K})$ & $(\AA)$ & $(\AA)$ & $(\AA)$ & $\begin{array}{c}\gamma \\
\left({ }^{\circ}\right)\end{array}$ & $\left(\AA^{3}\right)$ \\
\hline $120.0(5)$ & $3.76837(5)$ & $3.29445(4)$ & $7.89934(5)$ & 90 & $98.068(3)$ \\
$20.0(5)$ & $3.76745(6)$ & $3.28965(5)$ & $7.89228(14)$ & $90.212(1)$ & $97.813(3)$ \\
$3.2(5)$ & $3.76749(8)$ & $3.28950(10)$ & $7.89257(27)$ & $90.212(2)$ & $97.813(5)$ \\
\hline \hline
\end{tabular}



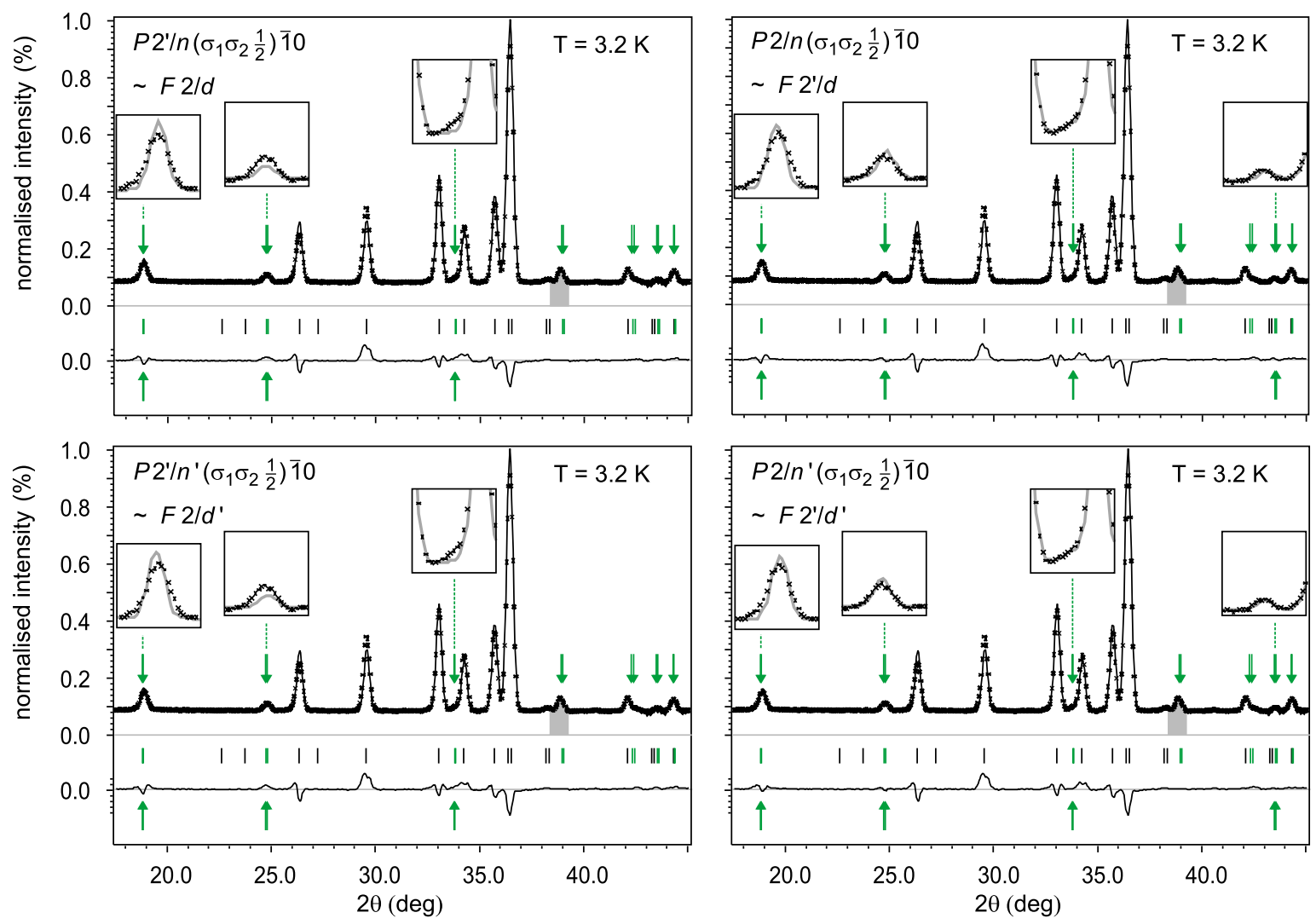

FIG. 3. (Color online) Rietveld plots of the powder neutron-diffraction patterns of VOCl at $T=3.2 \mathrm{~K}$ for refinements in four different magnetic superspace groups (magnetic space groups of the supercell are indicated). Observed (crosses), calculated (solid line), and difference intensity profiles are shown. The positions of the structural Bragg peaks are indicated by black vertical bars, the positions of the magnetic reflections by gray (green online) bars and the latter are highlighted by gray (green) arrows. The gray regions were excluded from refinement. Insets give expanded views around selected magnetic Bragg reflections.

values reflect the fact that the nuclear structures are identical between these models and that they are responsible for almost all of the diffracted intensity. Inspection of the Rietveld plots shows that the magnetic superlattice peaks (arrows in Fig. 3) are very well described in $F 2^{\prime} / d^{\prime}$ and $F 2^{\prime} / d$. In contrast, $F 2 / d$ and $F 2 / d^{\prime}$ do not account for the magnetic Bragg reflection at $2 \theta=33.8^{\circ}$, and they do not provide a good description of the magnetic reflection at $2 \theta=24.8^{\circ}$. This is commensurate with the slightly higher profile $R$ values of these models.

The magnetic superlattice peak at $2 \theta \simeq 43.5^{\circ}$ is well described in $F 2^{\prime} / d^{\prime}$ while in $F 2^{\prime} / d$ it appears shifted by an amount equal to the monoclinic split of the reflections $(1, \pm 3,1)$. Also for the magnetic superlattice peak at $2 \theta$ $=24.8^{\circ}$ the fit in $F 2^{\prime} / d^{\prime}$ appears slightly better than in $F 2^{\prime} / d$ but the magnetic peaks at $2 \theta=33.8^{\circ}$ and $2 \theta=18.9^{\circ}$ are equally well described in both symmetries. Combined with the fact that also $R_{p}$ and $R_{\text {Bragg }}$ are lower for $F 2^{\prime} / d^{\prime}$ than for $F 2^{\prime} / d$, we conclude that our data show a preference toward the symmetry $F 2^{\prime} / d^{\prime}$.

The temperature dependence of the anisotropic magnetic susceptibility indicates that ordered magnetic moments are parallel or nearly parallel to a. ${ }^{9}$ This is also reflected in the refined magnetic superstructure with $M_{x}=1.31(6)$ and $M_{y}=$ -0.04(11) for magnetic space group $F 2^{\prime} / d^{\prime}$ and $M_{x}$ $=1.29(11)$ and $M_{y}=0.05(11)$ for $F 2^{\prime} / d$. Therefore, we have repeated the refinements in $F 2^{\prime} / d^{\prime}$ and $F 2^{\prime} / d$ with the additional restriction $M_{y}=0$ [Eq. (2)]. Except for this restriction, the refined model and $R$ values are equal to the cases of free refinements of the direction of the magnetic moment, with $M_{x}=1.32(4), R_{p}=0.0507$, and $R_{\text {Bragg }}=0.0707$ for $F 2^{\prime} / d^{\prime}$, and $M_{x}=1.31(4), R_{p}=0.0510$, and $R_{\text {Bragg }}=0.0712$ for $F 2^{\prime} / d$. These refinements confirm the preference of a model with magnetic space group $F 2^{\prime} / d^{\prime}$ over a model with $F 2^{\prime} / d$.

The magnetic superstructure thus is described by a $2 \mathbf{a}$ $\times 2 \mathbf{b} \times 2 \mathbf{c}$ supercell with $F 2^{\prime} / d^{\prime}$ or $F 2^{\prime} / d$ symmetries. For $F 2^{\prime} / d^{\prime}$ the refined magnetic moment of $\mathrm{V} 1$ at $\left[\frac{1}{8}, \frac{3}{8}, 0.0577(17)\right]$ is $\mathbf{M}(\mathrm{V} 1)=[1.32(4), 0,0]$ with a magnitude of $1.32 \mu_{\mathrm{B}}$. For $F 2^{\prime} / d$ the refined magnetic moment of $\mathrm{V} 1$ is $\mathbf{M}(\mathrm{V} 1)=[1.31(4), 0,0]$ with a magnitude of $1.31 \mu_{\mathrm{B}}$. The two symmetries lead to similar models which form stripes of ferromagnetic order and stripes of antiferromagnetic order along the $[1 \pm 10]$ directions of the pseudoorthorhombic unit cell (Fig. 4).

The superspace symmetry ensures perfect antiferromagnetic order with magnetic moments of equal magnitudes on all vanadium atoms. The symmetry of the magnetic supercell would allow for independent directions and magnitudes of the moments on the two independent vanadium atoms (red and blue arrows in Fig. 4). The lowering of the symmetry at the phase transition from orthorhombic to monoclinic leads to twinning of the low-temperature structure. The two do- 

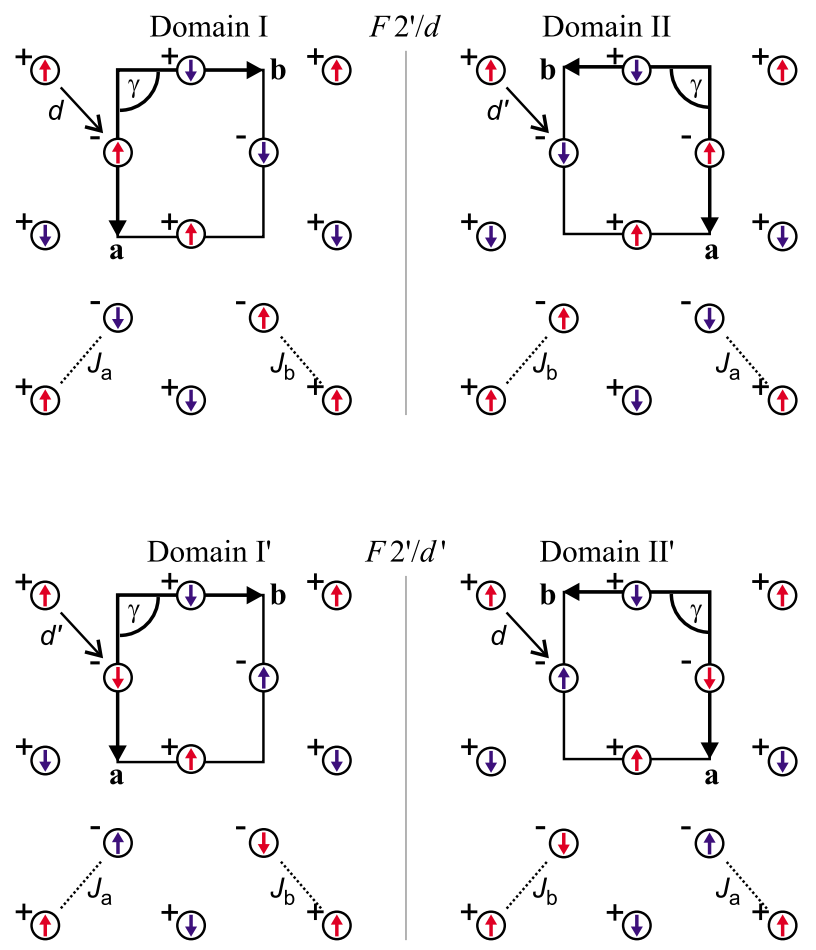

FIG. 4. (Color online) Schematic representation of the magnetic superstructure of $\mathrm{VOCl}$. For each of the models $F 2^{\prime} / d$ and $F 2^{\prime} / d^{\prime}$ the two domains are related by a mirror perpendicular to $\mathbf{b}^{*}$. Depicted are the vanadium atoms of one double layer $\mathrm{V}_{2} \mathrm{O}_{2}$ (compare Fig. 1 in Ref. 3) along with their magnetic moments for the two crystallographically independent atoms (red and blue arrows online, respectively). Atoms with coordinates $\left(0, \frac{1}{2},+z\right)$ and $\left(\frac{1}{2}, 0,-z\right)$ are indicated by "+" and "-," respectively.

mains are schematically drawn in Fig. 4. This twinning provides an alternative explanation for the observations which Wiedenmann et al. ${ }^{9}$ tried to explain by a coexistence of two phases.

\section{DISCUSSION}

The magnetic superstructure below $T_{\mathrm{N}}=80.3(3) \mathrm{K}$ has been determined as an antiferromagnetic ordering of monoclinic symmetry of the magnetic moments on the vanadium atoms (Fig. 2). This model is essentially different from the two-phase model with orthorhombic lattice symmetries proposed by Wiedenmann et al. ${ }^{9}$ Unequivocal evidence for monoclinic symmetry is given by the observation of split Bragg peaks both in single-crystal $\mathrm{x}$-ray diffraction and in powder-neutron diffraction (Sec. II).

A remarkable feature is that a structural distortion does not accompany the monoclinic lattice distortion. Positional and thermal parameters of the atoms obey the Pmmn symmetry of the small unit cell at all temperatures. For both single-crystal x-ray diffraction and powder-neutron diffraction, an improvement of the fit to the data could not be obtained by a further reduction in the nuclear symmetry together with the accompanying relaxation of structural parameters away from orthorhombic symmetry. In turn, this feature implies that the driving force for the monoclinic lat- tice distortion is exclusively provided by the magnetic interactions. In this respect $\mathrm{VOCl}$ differs from $\mathrm{TiOCl}$, in which magnetic order is accompanied by structural distortions. ${ }^{4}$

The compounds $M \mathrm{OX}(M=\mathrm{Ti}, \mathrm{V}, \mathrm{Cr}$, and $\mathrm{Fe}$, and $X=\mathrm{Cl}$ and $\mathrm{Br}$ ) have a common topology and a common symmetry at the metal site. As a working hypothesis we therefore assume an equal hierarchy of $3 d$ orbital energies. For TiOCl, calculated electronic band structures have been reported at various levels of theory. ${ }^{8,17,18}$ It has been found that the single-valence electron of $\mathrm{Ti}^{3+}$ occupies the $3 d_{x^{2}-y^{2}}$ orbitalfor $x$ along $\mathbf{b}, y$ along $\mathbf{c}$, and $z$ along $\mathbf{a}-$ that is responsible for antiferromagnetic coupling by direct exchange between neighboring $\mathrm{Ti}$ atoms along $\mathbf{b}$. The orbital next higher in energy is $3 d_{x z}{ }^{8,17,18}$ This orbital is empty in TiOCl. Here we assume that the two valence electrons of $\mathrm{V}^{3+}$ will occupy the $3 d_{x^{2}-y^{2}}$ and $3 d_{x z}$ orbitals. This then explains the observed antiferromagnetic order along b. Furthermore, it explains a strong, antiferromagnetic magnetic coupling between neighboring chains of vanadium atoms as governed by direct exchange involving the electron in the $3 d_{x z}$ orbital.

The monoclinic magnetic superstructure comprises antiferromagnetic order on the previously recognized chains of vanadium atoms along $\mathbf{b}$. Magnetic coupling between neighboring chains is frustrated on the orthorhombic lattice because couplings of equal magnitudes exists between neighboring vanadium atoms $\left(\frac{1}{2}, \frac{1}{2}, 0.115\right)$ apart (exchange constant $J_{\mathrm{b}}$ in Fig. 4) and between neighboring vanadium atoms $\left(\frac{1}{2},-\frac{1}{2}, 0.115\right)$ apart (exchange constant $J_{\mathrm{a}}$ ). For $\gamma$ $=90^{\circ} J_{\mathrm{a}}=J_{\mathrm{b}}$ and the two models $F 2^{\prime} / d$ and $F 2^{\prime} / d^{\prime}$ describe identical magnetic superstructures. The monoclinic lattice distortion lifts this degeneracy through minor modifications to the bond angles and interatomic distances. For $\gamma$ $=90.21^{\circ}>90^{\circ}$ - as employed in the present analysis - the bond $\left(\frac{1}{2}, \frac{1}{2}, 0.115\right)$ is slightly shorter than the bond $\left(\frac{1}{2}\right.$, $\left.-\frac{1}{2}, 0.115\right)$, implying $J_{\mathrm{b}} \neq J_{\mathrm{a}}$. Order according to $F 2^{\prime} / d$ occurs if $J_{\mathrm{b}}>J_{\mathrm{a}}$ while order according to $F 2^{\prime} / d^{\prime}$ requires $J_{\mathrm{b}}$ $<J_{\mathrm{a}}$. (Antiferromagnetic coupling is described by negative exchange parameters.) Both antiferromagnetic and ferromagnetic interchain coupling can thus lead to either of the two models, depending on the effect of the lattice distortion on the magnitudes of the exchange constants.

Our data give preference to magnetic symmetry $F 2^{\prime} / d^{\prime}$. Nevertheless, both models displayed in Fig. 4 share important features. Magnetic moments on vanadium atoms have the common direction along the a axis, parallel to the layers [Eq. (2)]. They form chains of antiferromagnetic order along $\mathbf{a}, \mathbf{b}$, and one of the directions $\frac{1}{2} \mathbf{a} \pm \frac{1}{2} \mathbf{b}$. The other diagonal direction then provides ferromagnetic chains. The difference between the two models is that antiferromagnetic order exists along the shortest bonds in $F 2^{\prime} / d^{\prime}$ while antiferromagnetic order exists along the longer of the two shortest bonds in $F 2^{\prime} / d$. In both cases ferromagnetic order exists on the other diagonal chains along $\left(\frac{1}{2}, \mp \frac{1}{2}, 0.115\right)$. This implies that both antiferromagnetic and ferromagnetic interchain couplings are compatible with the observed magnetic superstructure. The structure $F 2^{\prime} / d^{\prime}$ is the correct structure for ferromagnetic coupling with $\left|J_{\mathrm{b}}\right|<\left|J_{\mathrm{a}}\right|$ and for antiferromagnetic coupling with $\left|J_{\mathrm{b}}\right|>\left|J_{\mathrm{a}}\right| ; F 2^{\prime} / d$ would occur in the opposite cases (Fig. 4). 
The observed magnetic superstructure according to $F 2^{\prime} / d^{\prime}$ is the stable superstructure if the interactions between neighboring vanadium atoms are antiferromagnetic and stronger for the shorter $\mathrm{V}-\mathrm{V}$ bond-thus reflecting direct exchange interactions along the shortest $\mathrm{V}-\mathrm{V}$ bond of the structure (Fig. 4). Pursuing this interpretation further, one can assume that the magnetic exchange has both antiferromagnetic contributions (e.g., direct exchange of the $3 d_{x z}$ electron) and ferromagnetic contributions (e.g., superexchange involving the $3 d_{x^{2}-y^{2}}$ electron). ${ }^{18}$ It is not unreasonable to assume that the antiferromagnetic interactions are enhanced along the shorter $\mathrm{V}-\mathrm{V}$ bond while the ferromagnetic superexchange interactions are enhanced along the longer $\mathrm{V}-\mathrm{V}$ bond. Both ferromagnetic and antiferromagnetic contributions to the exchange coupling between chains then stabilize the observed monoclinic magnetic superstructure.

After completion of the present work we became aware of a preprint by Komarek et al. that has now been published. ${ }^{19}$ Komarek et al. $^{19}$ and the present work agree on the monoclinic lattice distortion and the magnetic superstructure of VOCl. The present work and Ref. 19 employ entirely different methods of structural analysis, which allowed us to conclude that the confinement of the magnetic moment to the a lattice direction is not the result of symmetry restrictions. An important feature noticed in the present work is that the observed magnetic superstructure is in agreement with both ferromagnetic and antiferromagnetic interchain interactions. Band structure calculations are required to establish the nature of the interchain interactions.

\section{CONCLUSIONS}

We have found that the development of magnetic order in $\mathrm{VOCl}$ below $T_{\mathrm{N}}$ is accompanied by a monoclinic lattice distortion. The lattice distortion gradually increases on cooling, suggesting a second-order phase transition, although the critical exponent $\beta$ of $0.099(12)$ is smaller than expected from simple theories. It has been shown by $\mathrm{x}$-ray diffraction that the lattice distortion is not accompanied by a structural distortion. Thus the driving force for the lattice distortion might be of pure magnetic origin.

Given antiferromagnetic coupling along b, both ferromagnetic and antiferromagnetic couplings between neighboring chains can explain the observed magnetic superstructure in VOCl. The ordered magnetic moments are parallel to the lattice direction $\mathbf{a}$, in agreement with the temperature dependence of the magnetic susceptibility. The magnetic superstructure obeys restrictions according to a magnetic superspace group thus reflecting magnetic order according to a single normal mode. The magnetic space group $F 2^{\prime} / d^{\prime}$ (standard setting $C 2^{\prime} / c^{\prime}$ ) (Ref. 15) allows a nonzero component of the magnetic moment of $\mathrm{V}$ along $\mathbf{b}$, which is, however, not found experimentally.

Magnetic order is stabilized by the monoclinic lattice distortion. The observed magnetic superstructure is in agreement with both ferromagnetic and antiferromagnetic interchain interactions. It is stabilized by antiferromagnetic contributions to the exchange interactions that are enhanced for the shorter of the two shortest $\mathrm{V}-\mathrm{V}$ bonds and by ferromagnetic contributions that are enhanced for the longer of the two shortest $\mathrm{V}-\mathrm{V}$ bonds. Theory should provide the character and relative magnitudes of the interchain exchange constants.

\section{ACKNOWLEDGMENTS}

Single crystals and powder sample were grown by A. Suttner. Experimental facilities and beam time were provided by Hasylab/DESY (beamline D3) and by FRM II/TUM (instrument SPODI). The authors gratefully acknowledge Vaclav Petricek and Michal Dusek for their help and assistance while refining the magnetic structures with JANA2006 and the German Science Foundation (DFG) for financial support.

\footnotetext{
*smash@uni-bayreuth.de

${ }^{\dagger}$ Present address: Department of Material Science, Technical University of Darmstadt, 64287 Darmstadt, Germany.

${ }^{\ddagger}$ Present address: HASYLAB at DESY, 22603 Hamburg, Germany.

${ }^{1}$ A. Seidel, C. A. Marianetti, F. C. Chou, G. Ceder, and P. A. Lee, Phys. Rev. B 67, 020405(R) (2003).

${ }^{2}$ A. Krimmel et al., Phys. Rev. B 73, 172413 (2006).

${ }^{3}$ A. Schönleber, S. van Smaalen, and L. Palatinus, Phys. Rev. B 73, 214410 (2006).

${ }^{4}$ M. Shaz, S. van Smaalen, L. Palatinus, M. Hoinkis, M. Klemm, S. Horn, and R. Claessen, Phys. Rev. B 71, 100405(R) (2005).

${ }^{5}$ S. van Smaalen, L. Palatinus, and A. Schönleber, Phys. Rev. B 72, 020105(R) (2005).

${ }^{6}$ P. Ehrlich and H.-J. Seifert, Z. Anorg. Allg. Chem. 301, 282 (1959).

${ }^{7}$ A. Haase and G. Brauer, Acta Crystallogr. B 31, 2521 (1975).

${ }^{8}$ T. Saha-Dasgupta, R. Valenti, H. Rosner, and C. Gros, Europhys. Lett. 67, 63 (2004).
}

${ }^{9}$ A. Wiedenmann, J. P. Vénien, P. Palvadeau, and J. RossatMignod, J. Phys. C 16, 5339 (1983).

${ }^{10}$ H. Schäfer, F. Wartenpfuhl, and E. Weise, Z. Anorg. Allg. Chem. 295, 268 (1958).

${ }^{11} \mathrm{X}$-ray diffraction at $T=20 \mathrm{~K}$ resulted in integrated intensities for 241 Bragg reflections, which were reduced to 112 unique observed reflections by the orthorhombic Laue symmetry $\mathrm{mmm}$. Absorption correction was applied. Structure refinements of the orthorhombic structure model in Pmmn resulted in a good fit with $R_{\text {obs }}=0.0370$ (2009).

${ }^{12}$ A. Schönleber, G. Shcheka, and S. van Smaalen, Phys. Rev. B 77, 094117 (2008).

${ }^{13}$ M. Hoelzel, A. Senyshyn, R. Gilles, H. Boysen, and H. Fuess, Neutron News 18, 23 (2007).

${ }^{14}$ V. Petricek, M. Dusek, and L. Palatinus, JANA2006. The Crystallographic Computing System (Institute of Physics, Praha, Czech Republic, 2006).

${ }^{15}$ D. B. Litvin, Acta Crystallogr. A 64, 419 (2008). 
${ }^{16}$ S. van Smaalen, Incommensurate Crystallography (Oxford University Press, Oxford, 2007).

${ }^{17}$ D. Fausti et al., Phys. Rev. B 75, 245114 (2007).

${ }^{18}$ Y.-Z. Zhang, H. O. Jeschke, and R. Valenti, Phys. Rev. B 78,
205104 (2008).

${ }^{19}$ A. C. Komarek, T. Taetz, M. T. Fernandez-Diaz, D. M. Trots, A. Möller, and M. Braden, Phys. Rev. B 79, 104425 (2009). 